\title{
Gesucht - der einfühlsame Unternehmer Erprobung praxisnaher Formen der Fachhochschulausbildung'
}

\author{
Prof. Dr. Bernd Wonneberger, Dipl.-Psych. Hans Könecke, Dr. Bernd Lehne, \\ Dipl.-Wirtschaftsing. (FH) Birgit Schmidt
}

\section{Problemstellung}

Eine zentrale Frage betriebswirtschaftlicher Ausbildung ist, wie man betriebliche Praxis stärker und besser in die (Fach-)Hochschulausbildung einbeziehen kann, um Studierende realitätsnäher mit den Problemen und Lösungsmethoden in den Betrieben vertraut zu machen. Dabei geht es auch um die Anwendung methodischer Instrumente und fachspezifischer Inhalte aus z. B. im Grundstudium erworbenen Wissens und Erkenntnisse betriebswirtschaftlicher Theorie auf die betriebliche Praxis, mit der nicht erst der Diplom-Betriebswirt (FH) oder Diplom-Kaufmann (FH) in voller Schärfe und abrupt nach dem Studium konfrontiert werden sollte. Für viele, die allein prüfungsbezogen gelernt haben, stellt sich dann heraus, daß sie ihr akkumuliertes Wissen nicht auf betriebliche Probleme im Beschaffungswesen, Rechnungswesen, Controlling oder Marketing umzusetzen vermögen und lange brauchen, sich in der betrieblichen Praxis zurechtzufinden. Es geht aber nicht allein um den Praxisschock oder die bekannte Erkenntnis, das betriebswirtschaftliche Studium hat mir zu wenig gebracht. Andere, die eine kaufmännische Lehre im Betrieb und gezielte betriebswirtschaftliche Weiterbildung neben der Berufstätigkeit betrieben haben, schaffen den Aufstieg häufig schneller und werden Fach(Hoch)schulabsolventen vorgezogen.

Betriebswirtschaftslehre - hier speziell Marketing und internationales Marketing - ist eine Realwissenschaft. Nichts wäre fataler für die zu qualifizierenden Studenten und die diese Qualifikation abnehmenden Betriebe, als daß sich die zwischen Theorie und Praxis auftuenden Gräben nicht verringerten. Da wir den Qualifikationsbegriff nicht eng sehen wollen - ein späterer Manager ohne ausreichende Allgemeinbildung ist undenkbar -, gehört die Allgemeinbildung und der Blick auf andere Wissenschaftsdisziplinen dazu.

In den folgenden Ausführungen beschränken wir uns auf die Frage einer besseren Einbeziehung der Praxis in die Ausbildung vor allem von Studenten im Hauptstudium. Es geht konkret um die Verbesserung des Projektstudiums.

\section{Was sind aktive Lernformen und was sollen sie bewirken?}

Die Lernforschung 2 hat seit mehr als einem Jahrzehnt auf den Nutzen aktiver Lenrformen hingewiesen. Was sind aktive Lernformen? In der Stärke der Ausprägung abgestuft kann man pragmatisch unterscheiden zwischen:
1. Beispielen: Beispiele, die in die Vorlesung einflochten werden, um an ihnen einen Sachverhalt zu demonstrieren, sind die schwächste Form des aktiven Lernens.

2. Fallbeispiele aus Büchern: Eine stärkere Form sind Fallbeispiele (z. B. aus Fallsammlungen oder der eigenen beruflichen Praxiserfahrung), an denen Studierende unter Anleitung des Dozenten ein Problem bearbeiten (Informationssammlung, Lösungsentwürfe etc). Diese enthält um so mehr aktive Lernelemente, je mehr Studenten in einzelnen Phasen aktiv tätig sind. Die vielfältigen Fallsammlungen in der Betriebswirtschaftslehre und ihren Funktionslehren wie z. B. Logistik, Kostenrechnung und Marketing sind eine gebräuchliche Form im Vermittlungsprozeß.

3. Unternehmensplanspiele: Diese stärkere Form aktiven Lernens hat einen größeren Stellenwert gewonnen. Sie wird z. B. als Blockseminar eine Woche am Semesteranfang oder Semesterende oder auch in der Praxisphase des Studiums angeboten. Wenn das Problem von mehreren Gruppen bearbeitet werden kann und Gruppen gegeneinander um die beste Lösung spielen, so erhöht dies meist die Motivation der Betroffenen, auch weil es mehr aktive Elemente enthält. Man kann die Situationen verändern und z. B. durch Zeitvorgaben Streß u. a. Faktoren einbauen, wie sie der betriebliche Alltag auch bietet. Der verbleibende Mangel oder das Defizit dabei ist, daß die menschliche Seite des Problems und seiner Lösung, der Unternehmer, fehlt. Es ist keine Ernstsituation.

4. Vergabe praktischer Probleme von Betrieben aufgrund der Erfahrungen der Dozenten und Studenten ohne explizite die Einbeziehung des Unternehmers oder eines realen Unternehmens in die Lösungserarbeitung, um z. B. im Marketing schöpferische, innovative Lösungen zu finden und dies zu trainieren.

5. Projektunterricht zu Problemen von Betrieben zusammen mit Unternehmensvertretern, bei KMU also Geschäftsführer, Marketingleiter etc.

Natürlich ließen sich noch Zwischenformen benennen, oder die vorgenommene Abstufung anders aufbauen. Sie alle fördern - anders als frontal vermittelte Vorlesungen mit passivem Aufnehmen und oft prüfungsbezogenem Lernen - ein Einbinden der Studierenden und aktives exemplarisches Lernen. Es geht ja bei der Ausbildung um Vermittlung fachlicher Qualifikationen und überfachlicher, (sozialer), extrafunktionaler Qualifikationen, die die von den Betrieben gewünschte Berufsqualifikation fördern und zunehmend von der Wirtschaft eingefordert werden. 
Daß ein Diplomand das Fachwissen und den Lernprozeß beherrscht, ist die Basis, reicht aber bei weitem nicht aus. Jeder Personalchef oder Abteilungsleiter gewichtet außerdem nach Auftreten, Allgemeinbildung, Auslandsaufenthalt, sozialem, sportlichen, künstlerischem Engagement und was der Student/in neben dem Studium sonst noch so gemacht hat. Neben wichtigen fachlichen Fähigkeiten sind es vor allem die sogenannten extrafunktionalen, auch überfachlichen oder sozialen Qualifikationen (wie z. B. zielstrebiges Problemlösen, Teamfähigkeit, Kritikfähigkeit, Eigeninitiative), die seit mehr als 10 Jahren zunehmend als sogenannte „Schlüsselqualifikationen“ herausgestellt werden. Vielleicht sollte man besser sagen: der Erwerb guter fachlicher Qualifikationen ist eine Selbstverständlichkeit und wird von jedem Betrieb vorausgesetzt.

Die überfachlichen Qualifikationen machen die eigentliche „Würze“ des Bewerbers aus - auch für den späteren Vorgesetzten wie die Teammitglieder im Betrieb. Je besser sie beherrscht bzw. in das Verhalten des Studenten eingegangen sind, desto besser eignet er sich nicht nur fuir seine derzeitige Stelle, sondern es ist ein Schluissel für den Aufstieg. Nicht umsonst zielen viele Bewerbertests von Betrieben wesentlich auf den Teil extrafunktionaler Qualifikation ab. Wie sollen diese überfachlichen Qualifikationen vermittelt werden? Hier fachliche Qualifikation, dort überfachliche Qualifikation - es gab (und gibt) vielfach noch eine künstliche Trennung. Längere Zeit wurde die Frage diskutiert, ob extrafunktionale Qualifikation in Rhetorik-, Präsentationstrainings $u$. a. vermittelt werden sollten. Vor rund 10 Jahren wurde hierauf eine Zwischenantwort gegeben ( $\mathrm{z}$. B. Franke): Die überfachlichen Qualifikationen sollten beim fachlichen Lernen mit vermittelt werden und nicht additiv oder getrennt. Dazu eignet sich der Projektunterricht besonders.

Knüpft man an die Ausgangssituation (Beispiele, Fälle, Planspiele) und den angestrebten Qualifikationsfundus an, so wird offenkundig, wo Defizite auftreten: Es ist, pauschal gesagt, die Ernstsituation, die fehlt. Die Studierenden haben es mit einem „gespielten“ Unternehmen, nicht mit echten Geschäftsführern, Entscheidern, Vorgesetzten zu tun. Aber auch beim Unternehmer im Seminarraum fehlt der echte Betrieb als Rahmen.

Unser Forschungsprojekt zielte in seinem didaktischen Teil dahin, ein betriebswirtschaftliches Problem eines Unternehmens mit einem realen Geschäftsführer oder Marketingleiter von der Informationssuche bis hin zu Problemlösungen mit allen Unzulänglichkeiten, auch unter Streß etc., zu bearbeiten und dies in verschiedenen Konstellationen zu versuchen.

\section{Verschiedene Formen von Projekt- unterricht}

Wenn wir den Projektunterricht aufgreifen, dann sprechen wir von nichts neuem. Schon vor mehr als einem Jahrzehnt haben innovative Betriebe im dualen System der Berufsausbildung neue Wege des Projektlernens (vielfach zusammen mit dem Bundesinstitut für Berufsbildung in sogenannten Modellversuchen) erprobt: So erbauten Auszubildende z. B. einen Motorsegler von der Kiellegung bis zum Stapellauf. Sie erwarben dabei in zwei bis drei Jahren berufliche Kenntnisse und Fertigkeiten aller daran beteiligten Berufe. Es könnten viele andere Beispiele genannt werden. Überhaupt scheint, daß das seit einiger Zeit zunehmend harsch kritisierte duale Ausbildungssystem ${ }^{3}$ mit sogenannten „Leitbetrieben“ (z. B. SIEMENS, BASF, BAYER) experimentierfreudiger ist und die Diskussion über den Erwerb von fachlichen und überfachlichen Qualifikationen eher aufgenommen hat als das akademische Bildungssystem. Hier sind es eher die Berufs- und Arbeitspädagogik sowie die Wirtschaftspädagogik, die eine Vorreiterfunktion haben. Betriebswirtschaftslehre und Lerntheorie waren häufig zwei Welten und sind es vielfach auch heute noch.

Verfolgt heute zu unserem Thema die Printmedien, so liest man in der Tageszeitung von einem Kollegen, der mit seinen Hauptstudium-Studenten ein Controllingsystem bei einem Betrieb eingefuihrt hat, von einem anderen, der mit seinem Studenten eine Prozeßkostenrechnung auf einen bestimmten Betrieb zugeschnitten hat u. a. Nicht zu vergessen sind die studentischen Unternehmensberatungen meist höherer Semester, die sich, gut gemischt mit verschiedenen Qualifikationen, auf dem Markt zu Einstiegspreisen zu etablieren beginnen.

Halten wir fest: Projektunterricht hat einen gewissen Stellenwert im Studium gewonnen und wird weiter zunehmen, weil seine Vorteile offenkundig sind.

Nun müssen wir verschiedene Formen des Projektunterrichts unterscheiden. Wir wollen dabei auf einen bestimmten Punkt abstellen und folgende Varianten formulieren: Entweder handelte es sich um ein konkretes betriebliches Problem, das im Hörsaal behandelt wird und bei dem der Dozent quasi den Geschäftsführer vertritt, oder die Studierenden (in der Regel im Hauptstudium) werden mit einem betrieblichen Problem konfrontiert und zugleich mit dem Verantwortlichen, sei es der Geschäftsführer oder der Marketingleiter, zusammengebracht und der Dozent übernimmt die Moderation, Planung und Steuerung. Bei der ersten Form kann man einen Betriebsbesuch in der Anfangsphase einplanen, damit die Studierenden konkrete Vorstellungen erhalten.

Wir haben beide Formen durchgeführt und wollen schwerpunktmäßig auf das zweite Modell abstellen, weil es bisher weniger erprobt worden ist.

\section{Erfahrungen aus dem Projektunterricht}

\subsection{Vorbereitung}

Ein betriebliches Projekt in vier Monaten mit Studierenden, Geschäftsführern und Unternehmen gut durchzuführen, setzt eine längere aufwendige Vorbereitung voraus. In der Regel sollte man das Unternehmen, vor allem den Geschäftsführer, vorher kennen. Wir konnten auf einen Bestand an mittelständischen Betrieben zurückgreifen, deren Geschäftsfuihrer wir in anderen Arbeitszusammenhängen (z. B. Beratung) kennengelernt hatten. 
Aber wir greifen auf Empfehlung des Technologie- und Weiterbildungszentrums unserer Fachhochschule auch auf andere Betriebe zurüick. In drei Projekten haben Studierende Betriebe vorgeschlagen, die sie kannten.

Das zu behandelnde Thema - ein betriebliches Marketingproblem - muß nicht nur reizvoll, sondern auch innerhalb eines Semesters lösbar sein. Es sei denn, das Projekt ließe sich fortsetzen (was selten geschieht, eher schließt sich eine Diplom-Arbeit an); außerdem sollte es für das Unternehmen ein Problem mit großer Dringlichkeit behandeln, damit der Betriebsinhaber/Geschäftsführer dahintersteht, sein Interesse artikuliert und zm Fortkommen durch entsprechende Informationen beiträgt. Wir haben den Studenten die Themen nebst Erläuterung vorher bekanntgegeben, damit sie sich damit vertraut machen konnten. Die Vorbereitung bezog sich auf Fragen wie Arbeitsorte des Unternehmens, Betriebszugänglichkeit und Übernahme der Projektkosten (Recherche-Kosten, Fahrtkosten, Kopierkosten u.a.).

Eine Schwierigkeit war in der Regel die zeitliche Verfügbarkeit des Geschäftsführers. So konnten die Projektsitzungen in der Hochschule meist nur am späten Nachmittag stattfinden, weil der Geschäftsfuihrer am Tag in der Regel kaum abkömmlich war.

Wichtig für die Festlegung des Schwierigkeitsgrades bei der Themen- und der Betriebssuche ist auch eine gute Kenntnis der Voraussetzungen der Studierenden. So waren z. B. „normale“ Marketing-Studenten überfordert, für eine Ingenieurbetrieb ein Konzept zur Markterschließung in Polen oder Indonesien zu bearbeiten, weil ihnen die theoretischen wie länderspezifischen Voraussetzungen oder die interkulturelle Kompetenz fehlten, also entsprechendes Wissen aus dem internationalen Marketing.

\subsection{Organisation des Projektunterrichts}

In der ersten Projektsitzung trafen sich alle Studenten und Betriebsvertreter. In der Regel waren der oder die Geschäftsführer anwesend, die ihre Betriebe und das Projekt aus betrieblicher Sicht ausführlich vorstellten und Fragen der Studierenden beantworteten.

Der persönliche Eindruck des Unternehmers bei den Studierenden, seine Persönlichkeit, seine Überzeugungskraft, sein Witz und Humor wie auch die Art des Eingehens auf Fragen der Studenten sind nicht hoch genug einzuschätzen. Aufgrund der Vorinformationen, der Themen und nicht zuletzt des „Sympathiegrades“ des Geschäftsfuihrers entschieden sich die Studierenden für das eine oder andere Projekt. Betriebsbesichtigungen und Problembesprechungen im Betrieb zu einem zweiten Termin gaben schnell den Ausschlag, wer sich für welches Problem endguiltig zusammenfand.

Diese Wahlmöglichkeiten halten wir für sehr wichtig, weil sie das fachliche Interesse der Studierenden stark berühren und dies auch ein ausschlaggebender Punkt für ihre Motivation ist. Es muß bei dem Problem im Vorfeld klar sein, wie das Ergebnis formal - aber in Elementen auch inhaltlich - aussehen soll (wovon die Geschäftsführer konkrete Wünsche und Vorstellungen haben). Vom Dozenten sollte der methodische Ablauf, also die
Schritte z. B. zu einem Marketing-Konzept oder zu einer Marktanalyse vorgedacht einschließlich des Zeitplans vorgedacht sein. Ob die für die Problemläsung benötigten Arbeitssitzungen im Betrieb, im Hörsaal oder an anderer Stelle durchgeführt werden, ist eine Frage der Zweckmäßigkeit und hängt von den konkreten Teilaufgaben ab. In der Regel waren es mehrere Lernorte.

In der zweiten Arbeitssitzung wurden (es war zugleich die Hausaufgabe) in einem Brainstorming-Verfahren Wege skizziert, die zu dem Ziel, dem erwarteteten Ergebnis führen könnten, und die Teilschritte zur Lösung des Problems erörtert. Auch stellte sich gleich die Frage, wer welchen Teil bearbeitet.

Die Projekte wurden so strukturiert, daß eine Zwischenpräsentation nach 1 1/2 Monaten und eine Abschlußpräsentation der Ergebnisse vor der Geschäftsführung vorgesehen waren.

In der Zwischenpräsentation mit Folien und Kurzreferaten, an der alle Projektgruppen verpflichtet waren teilzunehmen, stellten die Studierenden den bisher erarbeiteten Stand des Themas, offene und ungelöste Probleme bzw. Fragen sowie die geplante weitere Vorgehensweise dar.

Wichtig war auch, Anstöße und Hinweise für alle zu geben. Somit war der wöchentliche Termin der Projektzeit, in der Regel 30 Minuten je Gruppe, eine zentrale Anlaufstelle, um die Fortschritte zu sehen, bei Problemen zu helfen oder bei Institutionen zu vermitteln, die weiter helfen könnten (z. B. bei differenzierten Fragen im internationalen Marketing). Eine Anwesenheit des betrieblichen Verantwortlichen wurde - wenn erforderlich - vorher geklärt. Vieles wurde auch so geregelt, daß die Projektstudierenden bei betrieblichen Fragen den Verantwortlichen im Betrieb einfach anrufen konnten. Dennoch standen die Eigeninitiative und auch Eigenverantwortung, insbesondere beim oberen Semester, im Vordergrund.

Die Gruppengröße, die für die Arbeit benötigt wird, sollte vorher vom Dozenten festgelegt werden. Je nach Problemgröße und Schwierigkeitsgrad bilden 3 bis maximal 5 Studenten eine Arbeitsgruppe. Wir haben aber auch schon eine Gruppe von 12 Studenten gehabt, als wir einen Marktforschungsauftrag gewannen.

Es ist klar, daß es um die Überschaubarkeit und Leitung des Einzelnen in der Gruppe und eine möglichst gleichverteilte Arbeitsbelastung der Gruppenmitglieder geht. Wir wollen im folgenden auf einige ausgewählte Punkte eingehen, die wir für allgemein interessant halten.

\section{Zentrale Punkłe des Projekłunterrichts}

\subsection{Pädagogischer Unternehmer}

Die Persönlichkeit des Unternehmers/der Unternehmerin halten wir für einen zentralen Punkt für das Gelingen des Projektunterrichts. Man kann hier den „guten Draht" zu jungen Menschen, die Art, auf die Studenten einzugehen, „das Herüberbringen“ von Fachwissen, die Akzeptanz, daß Studenten auch andere Wege gehen oder erkennen lassen, Freude dabei haben, überzeugt sind, nennen, aber auch, daß ein für ihn brauchbares Er- 
gebnis herauskommt oder die eigene gute Erfahrung mit Diplomanden/Praktikanten. Das gibt es sicher immer nur in Grenzen. Mehrfach stießen wir schon bei der Planung des Projekts auf Skepsis, z. B. ob Studierende so etwas leisten können. Am leichtesten waren Geschäftsführer zu überzeugen, wenn man abgeschlossene Projektberichte vorlegte.

Als nicht geeignet erwies sich eine Unternehmerin, die die Studierenden bei der Suche nach betriebswirtschaftlich effizienten Vertriebswegen, Produktpräsentationen, kommunikativen Instrumenten und einer Preispolitik in eine bestimmte Richtung zu drängen versuchte, weil dadurch der Fluß weiterer brauchbarer Ideen erstickt wurde. Auch das bei bestimmten Lösungsvorchlägen immer wieder zu hörende Argument „Das können wir uns nicht leisten " wirkt kontraproduktiv (als ob nicht zusätzlichen Kosten zusätzliche Erträge, die in der Regel höher sind, gegenüber gestellt werden müssen). Hier ist der Dozent als Moderator und Mittler gefordert.

Übrigens ist es kaum eine Frage des Alters des Unternehmers/in, sondern der Aufgeschlossenheit, der Persönlichkeit und des Vertrauens in die Studierenden.

Ein weiterer wichtiger Punkt ist, daß Geschäftsführer von kleinen mittelständischen Unternehmen oft nur wenig Zeit haben, sich ihren betrieblichen Problemen ausreichend zu widmen und damit auch der Input an die Studenten unvollständig bleibt.

Der Leistungsheterogenität unter den Studenten könnte man ein eigenes Kapitel widmen. Sehr schnell wird deutlich, wer die Gruppe führt und die Arbeit verteilt. Nur wenn es gar nicht gelingen will, sollte der Dozent intervenieren und Arbeitsaufträge verteilen.

\subsection{Selbständiges Arbeiten an einem betrieblichen Problem}

Die Selbständigkeit des Arbeitens an einem konkreten betrieblichen Problem setzt ungeahnte Motivation und Energie frei. Obwohl der zu investierende Zeitaufwand meistens doppelt (und mehr) so hoch ist wie bei einer Übung, haben die Projektstudierenden fast immer den Einsatz gebracht. Sie hatten sich das Problem des Unternehmens zu ihrem eigenen gemacht und trieben Lösungswege bis zum fertigen Ergebnis voran.

Problematisch wird es eher, wenn viele Kollegen Projektunterricht durchfuihren, sich nicht abstimmen und die Arbeit im zweiten Drittel des Semesters zu Überlastungen bei den Studierenden führt.

Eine Kunst ist es, kompetente Informanten anzusprechen, die Recherchen zielgerichtet durchzuführen, keine Scheu zu haben, mit Verbänden, Forschungsinstituten und Professoren anderer Hochschulen Kontakt aufzunehmen.

Die Motivation der Studierenden wird aus mehreren Wurzeln gespeist:

- weil es für die meisten neu ist,

- weil es Spaß macht,

- weil sie mit einem Geschäftsfuihrer zusammenarbeiten, wozu sie sonst kaum Gelegenheit haben,
- weil sie ein realistisches, betriebliches Problem ihrer Wahl bearbeiten, wie später im Berufsleben,

- weil sie einen Spielraum der Arbeitsgestaltung haben,

- weil sie Anerkennung bei der Präsentation eines gelungenen Lösungsvorschlages erhalten.

Dies alles stärkt auch ihr Selbstwertgefühl und bringt sie weiter auf dem Wege zu ihrem künftigen Arbeitsplatz.

\subsection{Dozent als Moderator, Vermittler und Klammer}

Im Prozeß der Projektarbeit kommen auf den Dozenten Aufgaben zu, für die teilweise andere Qualifikationen als die üblichen erwartet werden. So hat seine Moderation eine wichtige Vermittlungsfunktion zwischen dem Unternehmer und den am Projekt arbeitenden Studenten. In „verfahrenen“ Situationen muß er das Knäuel entflechten, glätten und Wege weisen. Vielfach tauchen wie im richtigen Arbeitsleben auch (menschliche) Probleme auf. Damit die Studenten dennoch das Gefühl haben, daß es ihr Projekt ist, sollte sich der Dozent weitgehend zurückhalten, insbesondere, wenn das Projekt gut läuft. Die Unterstiitzung des Dozenten wird allerdings nötig, wenn die Studierenden bei ihren Informationserhebungen per Telefon, Fax, Brief, Internet - egal ob bei Wirtschaftsforschungsinstituten, Verbänden, Kammern, öffentliche Stellen, Betrieben - „abserviert“, vertröstet oder als Gesprächspartner nicht akzeptiert werden.

\subsection{Vermittlung fachlicher und überfachlicher Qualifikation}

Aktives Lernen ist durch die hier beschriebenen Projekte ohne Zweifel gegeben. Unsere Erfahrung war, daß die Studierenden mehr Sicherheit im Auftreten etc. gewannen. Auch läßt sich feststellen, daß vielfach die Diplomarbeit (z. B. über ein praktisches betriebliches Marketingproblem) nach solchen Projekterfahrungen selbständigen Arbeitens zielstrebiger bearbeitet und vom Ergebnis her brauchbarer und qualitativ besser wurde. Wir hatten eingangs darauf hingewiesen, daß mit dieser Art des Projektunterrichts sowohl fachliche wie überfachliche Qualifikationen vermittelt werden könnten. Dies läßt sich ohne Einschränkung bestätigen.

Wir möchten anmerken, daß unsere Studenten/-innen in einer eigenen Veranstaltung auch Rhetorik und Präsentationstechnik vermittelt bekommen.

Das schließlich präsentierte Ergebnis, das mit den Unternehmen diskutiert wurde, steht für die fachliche Seite. Die Art der Darstellung, Vermittlung, Argumentation und ggf. Verteidigung ist ein Teil der affektiven Komponente. Das Trainieren extrafunktionaler Qualifikationen der Studierenden erfolgte in der ganzen Zeit von der Problemanalyse und -bearbeitung über die Informationsphase bis hin zur Erstellung von Lösungsbausteinen-dies meist unter zeitlich begrenzten Bedingungen, wie sie auch im Betrieb bestehen. Nach einer solchen Projektarbeit hatten es die späteren Diplomanden merkbar leichter, praxisnahe Themen zu erörtern, weil sie mit der Arbeitsweise, der Methodik, den Problemen dem Unternehmen besser umzugehen gelernt hatten. 


\subsection{Brauchbarkeit der Ergebnisse}

Die Ergebnisse wurden am Semesterende von allen Gruppen den Unternehmern vorgestellt. Neben dem Ergebnisbericht (z. B. ausformuliertes Marketingkonzept) wurden sie mit Overheadprojektor, Flip Chart, Video u. a. Medien dargestellt (die Präsentation wurde mit rund $20 \%$ der Endnote bewertet). Es kam auch vor, daß Unternehmen uns zu einer Abschlußpräsentation vor der firmeneigenen Führungsmannschaft einluden. Die vorgetragenen Ergebnisse wurden diskutiert, insbesondere hinsichtlich der Umsetzbarkeit im Betrieb. Wir baten die Unternehmensrepräsentanten um eine Stellungnahme, insbesondere hinsichtlich der Brauchbarkeit der Lösung. Ein guter Indikator für die geleistete Arbeit war, wenn die Unternehmen auf uns zukamen und wir gefragt wurden, ob wir das Projekt z. B. im Rahmen einer Diplomarbeit weiterführen oder im nächsten Semester ein anderes Problem des Betriebes bearbeiten wollten.

\subsection{Umsetzung von Theorie in Praxis}

Ein Ziel des Projektunterrichts ist die Verbindung von betriebswirtschaftlicher Theorie und betrieblicher Praxis. Die Projektstudenten mußten erfahren, daß zu einigen betrieblichen Teilproblemen die betriebswirtschaftliche Theorie nichts sagt. Andere Fragen führten dazu, daß betriebswirtschaftliche „Theorieangebote“ fuir den konkreten Fall keine brauchbare Hilfe waren. Einige Fallsammlungen im Marketing haben den Nachteil, daß sie Fälle lehrbuchhaft darstellen und Probleme in ihrer Komplexität wesentlich reduzieren. Bei den behandelten Projekten herrscht deshalb ein gewisser Grad an Ungewißheit. Daß die Aufgaben nicht unlösbar sind, darauf sollte bei der Vorabsprache mit dem Unternehmen geachtet werden.

\section{Ausgewählte Probleme}

28 bis 34 Semesterwochenstunden sind für die Fachhochschulstudenten (mit zunehmender Erwerbstätigkeit nebenbei) zeitlich sehr belastend. Wenn also mehrere Kollegen gleichzeitig Projektunterricht für die oberen Semester anbieten, können die Studierenden zumindest am Semesterende in Engpäße geraten. Ein Ausweg wäre, daß mehrere, sich fachlich ergänzende Kollegen ein Problem aus unterschiedlicher Sicht betrachten und behandeln ließen, so daß die Projektstudenten an einem komplexen Problem mit mehreren Schwerpunkten arbeiten können ( z. B. unter Marketing-, organisatorischen, finanziellen und Kosten-Gesichtspunkten).

Wir haben selbst eine solche Kooperation probiert. Für ein Gründungsprojekt behandelten wir die Fragen der Vermarktung, während ein weiterer andere Dozent Themen der Unternehmensgründung vermittelte. Eine Ausnahme ist es sicher, wenn solche Projekte mit noch mehr Kollegen aus den verschiedenen Bereichen durchgefuihrt würde.

\section{Ausblick}

Projektunterricht stellt ein vielschichtiges Thema dar. Wir haben in unserem Artikel die Einbeziehung der Unternehmen und Unternehmer in den Projektunterricht besonders beleuchtet. Ausgangspunkt aller dieser Überlegungen war, mehr Praxisnähe in die Lehre zu holen und die Studierenden so besser auf ihre zukünftige Berufsrolle vorzubereiten. Wir haben darauf hingewiesen, daß es nicht allein auf das reine, meist überschätzte Fachwissen ankommt.

Die zu vermittelnden „Schlüsselqualifikationen“ reichen weit über die fachliche Ebene hinaus. Seit seit 10 Jahren ist die berufliche Lerntheorie gut gemanagter Betriebe der Meinung, daß die extrafunktionalen überfachlichen Qualifikationen mindestens den gleichen Stellenwert haben. Jeder Personalchef stellt heute nach formaler Prüfung der Fachkompetenz auf die sozialen Qualifikationen ab, wenn er neue Mitarbeiter sucht.

Wir sind der Meinung, daß überfachliche Qualifikationen nicht gesondert, sondern zusammen mit den fachlichen Qualifikationen vermittelt werden sollten. Auch deshalb ist auch der Projektunterricht stark auf dem Vormarsch; an Universitäten in den USA, in Großbritannien oder Holland ist dies schon die herrschende Lehrform.

In unserem Forschungsprojekt haben wir zwar nur eine Variante des Projektunterrichts erprobt, sind aber bereits hier zu Ergebnissen gekommen, die uns ermutigen, in diesem Sinne weiter zu machen.

\section{Fußnoten}

1 Teil des Forschungsprojektes: Markterschließung in Ost- und Südosteuropa für kleine und mittlere Unternehmen (KMU) in betrieblicher Kooperation und Erprobung praxisnaher Methoden der Fachhochschulausbildung.

2 vgl. diverse Studien und Modelversuche des Bundesinstituts für Berufsbildung Berlin-Bonn u.a. Franke, Guido; Kult, Konrad; Selka, Reinhard; Buck, Bernhard; Alt, Christel u.v.a.

3 vgl. u. a. Wonneberger, Bernd: Fehlende Lehrstellen und Fehlqualifizierung als Probleme des dualen Systems. Frankfurt a. M. 1993 (Peter Lang)

\section{Verfassser}

\section{Prof. Dr. Bernd Wonneberger}

Dipl.-Psych. Hans Könecke

Dr. Bernd Lehne

Dipl.-Wirtschaftsing. (FH) Birgit Schmidt

Technische Fachhochschule Wildau

Institut für internationales Marketing und

Unternehmensführung

Bahnhofstraße

15745 Wildau

Tel. +49 (0) 3375 - 508325

Fax +49 (0) $3375-508388$ 